\section{A young female with recurrent syncope}

A 23-year-old woman presented with multiple episodes of palpitations and loss of consciousness within a $48 \mathrm{~h}$ period. She gave a history of similar episodes since childhood and had been previously diagnosed as suffering from epilepsy. While being monitored in the hospital, she was found to have rapid polymorphic ventricular tachycardia (PVT) during the episodes. She required repeated cardioversion as the arrhythmia recurred despite administration of intravenous amiodarone and lignocaine. Prolonged monitoring showed frequent premature ventricular complexes (PVC) (figure 1A), couplets and short runs of ventricular tachycardia (figure 1B) and longer runs of ventricular tachycardia, which were associated with her clinical symptoms (figure 1C). Echocardiography showed no structural abnormalities. There was a history of sudden unexplained death in two younger siblings.

\section{QUESTION}

What is the most likely diagnosis in this patient?

A. Long QT syndrome (LQTS) with torsades de pointes (TdP)

B. Catecholaminergic polymorphic ventricular tachycardia (CPVT)

C. Short-coupled variant of TdP

D. Epilepsy with movement artefact

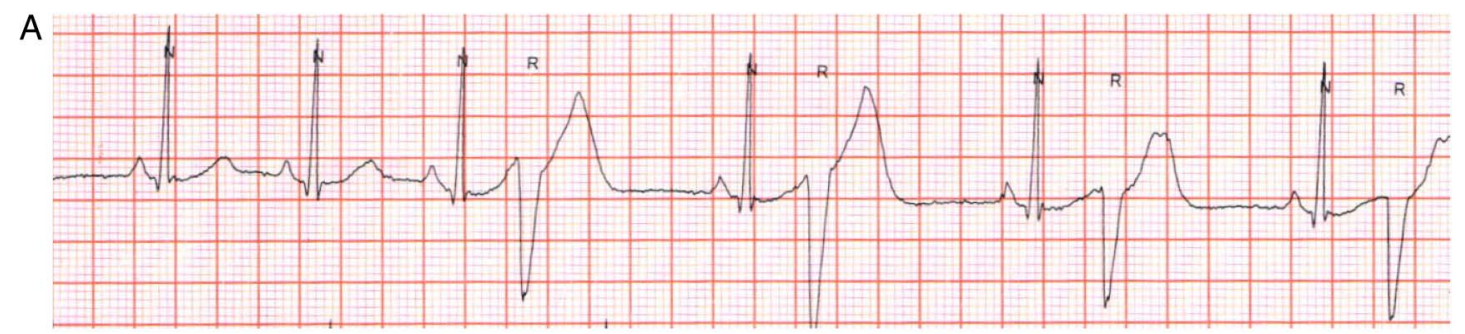

$\mathrm{B}$



C



Figure 1 Single-lead ECG from lead II recorded during long-term monitoring. Panels $A, B$ and $C$ show a short segment of recording from different times. N, 'Normal'; R, 'Ventricular ectopic'; V, 'Ventricular ectopic on T wave (R on T)'. 


\section{ANSWER: C}

Short-coupled variant of TdP

PVT with a gradual change in polarity as seen in panel 3 is described as TdP. This is classically and most commonly associated with prolongation of the QT interval due to congenital LQTS or acquired long QT. As the QT interval is normal during the sinus beats (QT $380 \mathrm{~ms}$, QTc $450 \mathrm{~ms}$ ), option A is wrong.

In CPVT, ventricular tachycardia is catecholamine dependent and is typically induced by exercise or emotional stress. The characteristic arrhythmia is bidirectional ventricular tachycardia, although PVT may also be seen.

Especially in long-term recordings, artefact has to be considered as a cause of rapid and irregular rhythms. Artefact is usually not associated with symptoms and uninterrupted normal QRS complexes can be seen within the artefact. Absence of these rules out this diagnosis.

In classical TdP associated with LQTS, the coupling interval of the first complex of the tachycardia run is long (>600 ms). In this patient, the coupling interval of the PVC or the first beat of PVT is very short, about $250 \mathrm{~ms}$. Short-coupled TdP is a rare variant of $\mathrm{TdP}$ with an extremely short coupling interval (almost always $<300 \mathrm{~ms}$ ) of the initial beat and a normal QT interval. ${ }^{1}$ It is a malignant disease with high mortality and occurs in healthy young individuals with a structurally normal heart. One-third of patients have a family history of sudden cardiac death.

Class I antiarrhythmics, $\beta$-blockers and amiodarone are all ineffective. Verapamil, an inhibitor of slow inward calcium current is the only drug with some efficacy to suppress this arrhythmia. However, it does not lower the risk of sudden death. Therefore, Implantable Cardioverter Defibrillator (ICD) is recommended.
This patient was treated with verapamil $240 \mathrm{mg} /$ day in three divided doses and showed an immediate and dramatic cessation of the ventricular arrhythmias. She subsequently underwent implantation of an ICD. At follow-up after 6 months, she continues to be on verapamil and had two appropriate ICD discharges.

\section{Mahadeva Swamy B, ${ }^{1}$ Deepak Amalnathan, ${ }^{2}$ Raja J Selvaraj ${ }^{1}$}

${ }^{1}$ Department of Cardiology, Jawaharlal Institute of Postgraduate Medical Education and Research, Puducherry, India

${ }^{2}$ Department of Medicine, Jawaharlal Institute of Postgraduate Medical Education and Research, Puducherry, India

Correspondence to Dr Raja J Selvaraj, Department of Cardiology, Jawaharlal Institute of Postgraduate Medical Education and Research (JIPMER), Puducherry 605006, Puducherry, India; raja.selvaraj@jipmer.edu.in, rajajs@gmail.com

Contributors All the three authors were involved in the clinical management of the patient. MSB and RJS prepared and revised the manuscript for submission with assistance from DA.

Competing interests None declared.

Patient consent Obtained.

Provenance and peer review Not commissioned; internally peer reviewed.

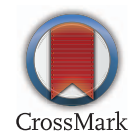

To cite Swamy B M, Amalnathan D, Selvaraj RJ. Heart Asia 2016;8:54-55. doi:10.1136/heartasia-2016-010732

Heart Asia 2016;8:54-55. doi:10.1136/heartasia-2016-010732

\section{REFERENCE}

1 Leenhardt A, Glaser E, Burguera M, et al. Short-coupled variant of torsade de pointes. A new electrocardiographic entity in the spectrum of idiopathic ventricular tachyarrhythmias. Circulation 1994;89:206-15. 